\title{
Analysis of cell proliferation in Drosophila wing imaginal discs using mosaic clones
}

\author{
T Dubatolova and L Omelyanchuk \\ Laboratory of Cell Cycle Genetics, Institute of Cytology and Genetics, Lavrent'eva 10, Novosibirsk 630090, Russia
}

\begin{abstract}
Experimental data on spatial and temporal distributions of mosaic clones in Drosophila wing imaginal disc were analyzed. Long-lived proliferation centers (PR1, PR2, and PR3) and areas with decreased proliferation activity were found in the notum region of the disc. Simulation of the growth kinetics of mosaic patches demonstrated that the cell cycle in proliferation centers PR2 and PR3 was shorter than the average cycle in the disc and in the center PR1. A nonrandom clustering of rapidly dividing cells was observed in the PR2, but not in the other cases. The reason why the cell-cycle duration and the clustering of dividing cells may not
\end{abstract}

coincide is discussed in terms of the recruitment of nondividing cells into the cell cycle. The simulation of the time course of the first and second moments of the size distribution of mosaic clones allowed the variance of cellcycle progression rates to be determined and demonstrated that a model with a continuous cell-cycle rates gave a better fit to the data than the transition probability model of Smith and Martin.

Heredity (2004) 92, 299-305, advance online publication, 21 January 2004; doi:10.1038/sj.hdy.6800408

Keywords: Drosophila; imaginal disc; mosaic clone; proliferation; growth

\section{Introduction}

The discovery of the compartment organization of Drosophila imaginal discs, which was carried out using mosaic clones, demonstrated the great utility of the study of mosaic clones in developmental biology (Garcia-Bellido et al, 1973). An equally important application of genetic mosaicism was the construction of the so-called fate maps (Garcia-Bellido and Marriam, 1969). In the fate mapping of blastoderm cell groups, a distance, defined as the probability of a mosaic patch boundary separating two structures, is used. The earliest data on the parameters of Drosophila cell cycle were also obtained through analyzing the growth of mosaic patches induced at different time points in development and recorded in the cuticle of adults (Garcia-Bellido and Merriam, 1971). In the mid-1990s, there was a revival in interest in mosaic clones in developmental research, as it was demonstrated that this type of analysis allowed proliferation centers, and the role of wing veins in their demarcation, to be detected (Gonzalez-Gaitan et al, 1994). In addition, the system \{hsFLP; Act-FRT-LacZ\}, allowing mosaic clones to be visualized at any moment during development, appeared; this allowed the directed cell allocation in the wing disc to be demonstrated (Milan et al, 1996b). The goal of the current work is to improve the analysis of mosaic clone distributions as a tool for research into cell proliferation.
Correspondence: L Omelyanchuk, Laboratory of Cell Cycle Genetics, Institute of Cytology and Genetics, Lavrent'eva 10, Novosibirsk 630090, Russia.E-mail: ome@bionet.nsc.ru

Received 2 December 2002; accepted 14 September 2003

\section{Materials and methods}

A Drosophila line carrying a $\mathrm{P}$ element with $\beta$-galactosidase reporter gene separated from constitutive actin promoter with a cassette of FRT sites, P\{Act5C(FRT. polyA)lacZ.nls1\} (Struhl and Basler, 1993), was obtained by courtesy of these authors. As the localization of the insertion was unknown, we localized it on the map of the polytene chromosomes, to the region $82 \mathrm{C}$. In this experiment, the vector Carnegie-20, containing the sequence of the $r y^{+}$gene, as does the construct $\mathrm{P}\{$ Act5C(FRT.polyA)lacZ.nls\}, was used as a hybridization probe. The probe was labeled using a Gibco BRL kit for nick-translation and hybridized to the preparation as described in Gell and Pardue (1971) at $37^{\circ} \mathrm{C}$.

The line $w$ hsFLP, carrying an inserted $\mathrm{P}$ element with the gene for FLP recombinase under the control of a heatshock promoter, was obtained by courtesy of Dr $S$ Nokkala, University of Turku (Finland). To stain the cells for the activity of reporter $\beta$-galactosidase, organs were isolated in Hank's solution, fixed for $20 \mathrm{~min}$ in $0.75 \%$ glutaraldehyde in $0.1 \mathrm{M}$ sodium-cacodylate buffer, and the fixer was washed off in PBS. The organs were then placed into the staining solution $(10 \mathrm{mM} \mathrm{NaH}$ $\mathrm{PO}_{4} \times 2 \mathrm{H}_{2} \mathrm{O} / \mathrm{Na}_{2} \mathrm{HPO}_{4} \times 2 \mathrm{H}_{2} \mathrm{O}$, pH $7.2 ; 150 \mathrm{mM} \mathrm{NaCl}$; $1 \mathrm{mM} \quad \mathrm{MgCl}_{2} \times 6 \mathrm{H}_{2} \mathrm{O} ; \quad 0.3 \%$ Triton X-100; $3.1 \mathrm{mM}$ $\mathrm{K}\left[\mathrm{Fe}^{\mathrm{II}}(\mathrm{CN})_{6}\right] ; 3.1 \mathrm{mM} \mathrm{K} \mathrm{K}_{3}\left[\mathrm{Fe}^{\mathrm{III}}(\mathrm{CN})_{6}\right]$; and $0.2 \% \quad \mathrm{X}$-gal) and kept overnight. To generate mosaic clones, \{Act5C.FRT.LacZ\} females were crossed with y $w$ hsFLP males. The resulting progeny were heat-shocked $(30 \mathrm{~min}$ at $37^{\circ} \mathrm{C}$ ) at the larval stage, and female larvae that immobilized on the tube walls for pupation were picked at different intervals. Therefore, the time between the clone induction and the end of third larval instar was recorded in each case. The mosaic clones produced were photographed using an NU-2 microscope equipped with 
a Kodak-290 digital camera at a resolution of $1440 \times 580$ pixel in a monochromatic light $(656 \mathrm{~nm})$, obtained using an interference filter. According to our measurements, the maximum absorption of the stained product in the reaction of X-gal and $\beta$-galactosidase is in this spectral region. The mosaic clones located at different depths were summed, because peripodial membrane cells, which are the source of some clones, are significantly lower in number than columnar epithelial ones.

Digital images of imaginal discs with stained clones were projected onto the map of wing imaginal disc (Figure 1 from Modollel and Campuzano, 1998). The authors are grateful to J Modollel for permission to use this figure in our work. The number of cells of each mosaic clone was estimated from its area in the digital image. The cell counts were verified by experiments involving the simultaneous staining of mosaic patches as described above, and staining nuclear DNA with Hoechst 33258 ( $1 \mathrm{mg} / \mathrm{ml}$ in PBS for $30 \mathrm{~s})$. The luminescence of nuclei was examined using an Olympus microscope. The mitotic index was calculated as the number of metaphases per 1000 cells.

Mitotic chromosome preparations used in the experiment to study the effect of heat shock on the cell cycle and the preparations for in situ hybridization were carried out as described in Omelyanchuk et al (1997).



Figure 1 Localization of one-cell clones in the wing imaginal disc (total data on all the time points).

\section{Results}

\section{Localized proliferation}

Mosaic clones were generated by heat shock at various time points before completion of the third larval instar. To determine the time of heat shock more precisely relative to the end of the third larval instar, larvae that crawled out onto the tube walls for pupation at various intervals after the heat shock were used. The data on the localization of one-cell clones induced at different time points of development are summarized in Figure 1. It is evident that these mosaic patches cover the entire disc in a uniform manner, that is, any variation with position in the disc in the efficiency with which mosaic clones are induced must be slight, at most.

The distribution of two-cell clones, that is, clones where a parental cell has progressed through one division after its induction is summarized in Figure 2. Comparison of the pattern with that in Figure 1 indicates that the distribution of dividing cells is less uniform areas of the clustering are evident in Figure 2 - along with rather large regions that have been free of cell divisions during the developmental period in question. These latter regions are predominantly localized to the future wing area and are likely to reflect the zone of nonproliferating cells (ZNC), localized on the dorsal/



Figure 2 Localization of two-cell clones in the wing imaginal disc (total data on all the time points). 
ventral $(\mathrm{D} / \mathrm{V})$ boundary of the wing (O'Brochta and Bryant, 1985; Johnston and Edgar, 1998).

To detect the regions with a long-term increased mitotic activity, we analyzed the distribution of clones containing two or more cells over the disc surface at different times of mosaic clone induction. For this purpose, the imaginal disc map on which was plotted localization of the clones was divided into square sectors with a linear size of $1 / 20$ of its largest axis and the number of clones was estimated in each square. The position of a square sector was sequentially shifted by $1 /$ 3 of its edge until the entire map of the disc had been included. The clone distribution was assumed to be Poisson with $\mu=m \times \mathrm{d} s / S$, where $m$ is the total number of clones induced at a particular developmental stage; $S$ is the area of disc map; and ds is the area of a square (ds/ $S=0.0055)$. In the squares displaying considerably higher counts of clones, $k$, than the average number of clones per square, the probability of this excess of clones appearing randomly was estimated as $P(k)=\left((\mu)^{k} \exp \right.$ $(-\mu)) / k !$. The squares displaying $P(k)<0.005$ were considered to contain nonrandomly large clusters of clones and are shown in Figure 3. The groups of overlapping squares were assumed to be long-lived proliferation centers. As a result of this analysis, three



Figure 3 Localization of long-lived proliferation centers in the wing imaginal disc. The square sectors containing 'nonrandom' clone clusters containing two or more cells are shown. Numbers indicate the time point when the cluster was observed; bold line and hatching, the border of proliferation center defined as an envelope including a group of overlapping square sectors. proliferation centers, designated as PR1, PR2, and PR3, were found.

Similarly, nonrandom clusters of clones with slowly dividing cells were detected. One- and two-cell clones induced $24-28 \mathrm{~h}$ (the maximal periods considered) before the end of the third larval instar were considered. No one-cell clones were found among those induced $28 \mathrm{~h}$ before the end of the third instar, suggesting that the number of imaginal disc cells leaving the cell cycle is rather small, if they do it at all. This also means that it is adequate to consider one-cell clones as the clones containing slowly proliferating cells. The square sections containing clusters of slowly dividing clones are shown in Figure 4. It is evident that the proliferation centers PR1 and PR2 are adjacent to patches of slowly dividing cells.

Projection of the map onto the map of presumptive rudiments of the wing imaginal disc (Bryant, 1975) demonstrates that the proliferation center PR2 is located between a pair of presumptive notopleural (NP) bristles and a pair of presumptive supraalar (SA) bristles. On the map of imaginal disc, the line between NP structures and the line between SA structures form an angle of $45^{\circ}$. However, these lines on the adult cuticle are almost parallel. This indicates that the role of the proliferation center PR2 and the overlapping center of slow prolifera-

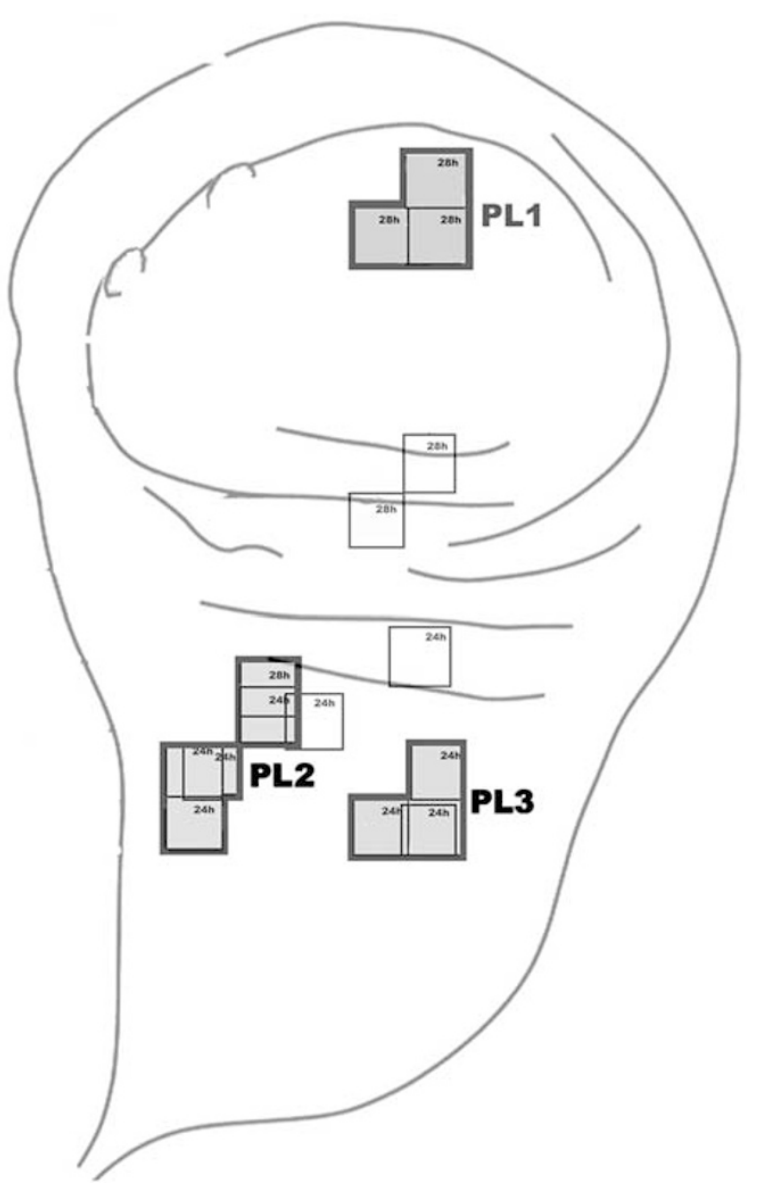

Figure 4 Localization of clusters of slowly dividing clones in the wing imaginal disc. Locations of nonrandom clusters of one- and two-cell clones are shown. Numbers indicate the time point when the cluster was observed; bold line and hatching, the border of overlapping square sectors. 
tion in rotating these structures relative to each other. We assume that the roles of PR1 and PR3 in turning the SA and postalar bristle (PA) structures are similar.

The proliferation centers PR1, PR2, and PR3 were then characterized according to the degree of clustering of rapidly dividing cell clones. For this purpose, the clones containing the highest cell numbers for the $12-28 \mathrm{~h}$ time points were plotted on the map of imaginal disc. The clones that are among the 5\% largest in the clone size distribution were considered to be large. The distribution pattern of the large clones relative to the proliferation centers in question is shown in Figure 5. The probabilities of the numbers of rapidly dividing clone clusters observed to appear in the regions PR1, PR2, and PR3 were calculated based on a Poisson distribution of the clones over the disc surface. The probabilities were $0.062,1.128 \times 10^{-7}$, and 0.073 , respectively. Thus, it is evident that the clustering of the rapidly dividing clones in PR2 is nonrandom, whereas the corresponding probabilities for PR1 and PR3 show no evidence of nonrandomness.

\section{Rate of cell division}

In this experiment, clones of different sizes were counted, as a function of the time when they were induced relative to the end of the third larval instar.

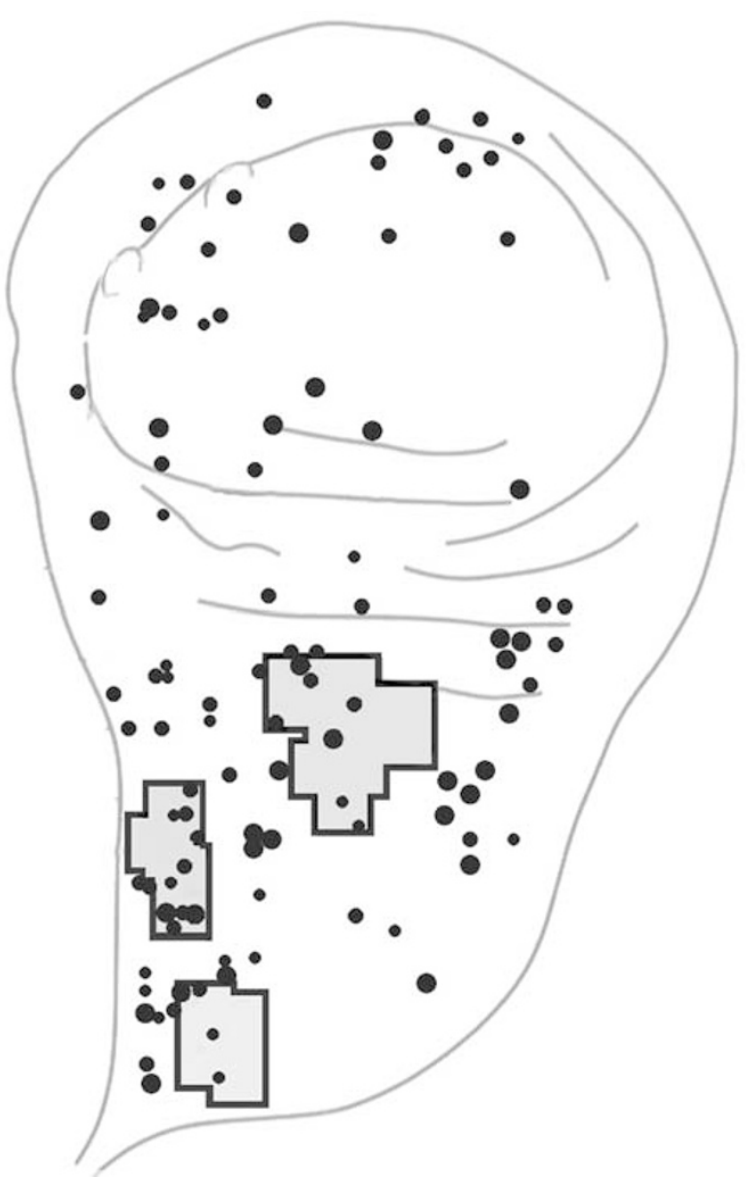

Figure 5 Localization of quickly dividing clones relative to the proliferation centers. Bold line and hatching indicate the proliferation centers; black circles, quickly dividing clones; and circle size, clone sizes (three cell, four to five cell, six to seven cell, and larger).
The relationship between the numbers of clones of different sizes and the time of induction is shown in Figure 6. It is evident that the maximal total count for all the clones, summing across the size classes is reached at $14 \mathrm{~h}$. Clearly, the rise of these curves results from the fact that certain time is required for protein synthesis, to detect the reporter $\beta$-galactosidase activity. This interpretation is in agreement with the data on growth time course of the clones (see below). There is another cause of the growth observed, namely, the activation of mitoses upon unblocking the cell cycle, which had been blocked by the heat shock used to induce the clones (see below).

The effect of heat shock on the time course of clone growth has rather a complex character (see below). Consequently, we searched for a region on the curves displaying an exponential growth of the average clone size, typical of a normal wing development (GarciaBellido and Merriam, 1971). For this purpose, an average size of the mosaic clone at each time point $M=M(t)$ was calculated from the weighted numbers of mosaic clones of different size classes:

$$
\begin{aligned}
& r_{i}(t)=N_{i}(t) / \Sigma N i(t) \\
& M=r_{1}+2 r_{2}+3 r_{3}+5 r_{4}+8 r_{5}+10 r_{6}
\end{aligned}
$$

where $r_{i}$ is the frequency of a clone of $i$-size class and 1, 2, $3,5,8,10$, respectively, are the average cell numbers within clones of a given size classes.

The resulting dependencies $M(t)$ for the entire disc; proliferation centers PR1, PR2, and PR3; and slow proliferation regions PL1, PL2, and PL3 were approximated by the curve $\exp \alpha\left(t-t_{0}\right)$. The parameters $\alpha$ and $t_{0}$ correspond to the cell generation time (1/cell-cycle duration) and to the time required for an exponential mode of clone growth to be reached (when the average clone size equals unity). These parameters were determined by computer fitting of the theoretical curve to experimental plots. The optimization results are listed in Table 1.

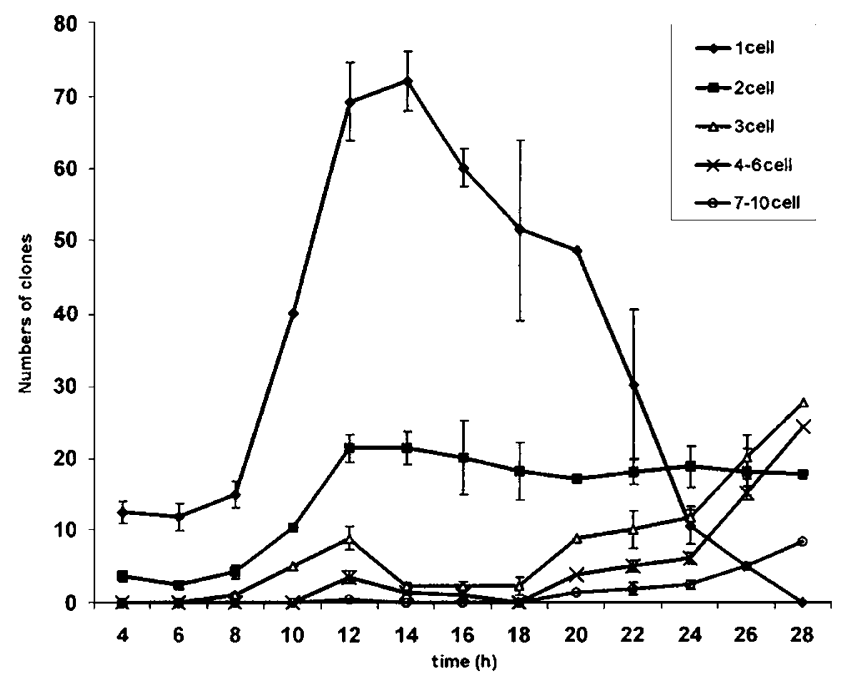

Figure 6 Dependences of the numbers of different-sized clones on the time point of clone induction. The ordinate, mean number of clones of particular size per disc; the abscissa, the time period between mosaic clone induction and end of the third larval instar. The clone size on the right of the plot. 
Table 1 Parameters of cell proliferation of the wing disc and its parts calculated by fitting theoretical curves to experimental plots covering $18-28 \mathrm{~h}$

\begin{tabular}{lcrrrr}
\hline & $\alpha(l / h)$ & $1 / \alpha(h)$ & $\mathrm{t}_{0}(h)$ & $\alpha_{\text {theor }}$ & $\alpha_{\max }$ \\
\hline Whole disc & 0.104 & 9.64 & 14.50 & 0.105 & 0.109 \\
PR1 & 0.103 & 9.66 & 12.95 & 0.102 & 0.109 \\
PR2 & 0.123 & 8.10 & 16.80 & 0.129 & 0.134 \\
PR3 & 0.114 & 8.77 & 12.51 & 0.114 & 0.118 \\
PL1 & 0.098 & 10.22 & 15.35 & & \\
PL2 & 0.108 & 9.24 & 15.60 & & \\
PL3 & 0.099 & 10.05 & 15.00 & & \\
\hline
\end{tabular}

The data listed demonstrate that the time required for reaching an exponential cell proliferation mode determined by the optimization in no case exceeds $18 \mathrm{~h}$, that is, the region of exponential growth was selected correctly. The time required for reaching a stationary growth mode corresponds to the time point when an inhibiting effect of heat shock on proliferation expires. It is evident that the cell generation times in the proliferation centers PR2 and PR3 are somewhat shorter compared to the average time for the disc; in the centers of slow proliferation, PL1, PL3, cell generation times are slightly longer than the entire disc average.

\section{Dispersion of cell-cycle rates}

Studies of the growth rates of mosaic patches and the rates of accumulation of BrdU label in, and disappearance from, wing imaginal disc chromosomes have demonstrated an intrinsic variation in the cell-cycle rate (Milan et al, 1996a; Tsuji and Tobari, 1980). However, the analyses performed in these works failed to show the range of cell-cycle rate variation in the tissues considered. The goal of this work was to determine the range of the rate variation based on the data on the time course of mosaic clone growth. In addition, we tried to answer the question as to whether the cell-cycle model of Smith and Martin (1973) was capable of an adequate description of the experimental data obtained.

As was demonstrated above, the exponential growth of mosaic clones in our experiments was observed within the time range of $18-28 \mathrm{~h}$. The mean size of mosaic clones (see above) was calculated as well as its mean square $M 2(t)$ (the designations are similar to those used above)

$$
M 2(t)=r_{1}+4 r_{2}+9 r_{3}+25 r_{4}+64 r_{5}+100 r_{6}
$$

The mean size of the clone growing with the rate $\alpha$ equals $N 1(t)=\exp (\alpha t)$ (the mean size equals unity at $t=0)$. The study of clone size variation in the clones induced in the wing disc at the end of second instar and the clones induced in the middle of third instar show an essential similarity in their distributions (Gonzalez-Gaitan et al, 1994), and give grounds for introducing a function $\rho(\alpha)$ - defined as the relative number of cells with proliferation rate $\alpha$, that is, at the start of exponential growth. The integration of this function between the maximal $\left(\alpha_{\max }\right)$ and minimal $\left(\alpha_{\min }\right)$ proliferation rate is assumed to give unity. The function is assumed to be constant over the studied time interval.

If a rate distribution $\rho(\alpha)$ exists, the mean clone size and its second moment are determined as

$$
\begin{aligned}
& N_{1}(t)=\int_{0}^{\infty} \exp (\alpha t) \rho(\alpha) \mathrm{d} \alpha \\
& N_{2}(t)=\int_{0}^{\infty}\left(N_{1}(t)-\exp (\alpha t)\right)^{2} \rho(\alpha) \mathrm{d} \alpha
\end{aligned}
$$

The normal distribution $\rho(\alpha)=\exp \left(\left(\alpha-\alpha_{0}\right)^{2} / D\right)$ truncated by the value which we call $\rho\left(\alpha_{\max }\right)$ and thereby deprived of infinite 'tails', and normalized to unity, was selected as a model distribution. The corresponding data from Table 1 were taken as the clone growth rates of the overall imaginal disc and proliferation centers; as the starting point of time count in $N_{1}(t)$ and $N_{2}(t)$, the $t_{0}$ value for the whole disc was taken from Table 1 . The best fitting of theoretical to experimental plots (Figure 7a and $b$ ) was observed for $D$ values exceeding $\alpha_{\max }$. The curve shape under these conditions ceases depending on $D$. Thus, the curves considered actually have two adjustment parameters $-\alpha$ and $\alpha_{\max }-$ for fitting the theoretical curve to experimental. The values obtained from these parameters are listed in the last columns of Table 1. It is evident that the fitting performed has no significant effect on these parameters.

In the transition probability model of Smith-Martin, the initial part of the cells generation time distribution (measured as the number of cells remaining in interphase, as a function of time in initially synchronized cell population, or alternatively the cells distribution over intermitotic time being revealed in a time-lapse cinematography experiments) is represented by a plateau (no cell exits the cell cycle before the time point $T_{0}$ ); then cells commence leaving the cell cycle according to the time

a

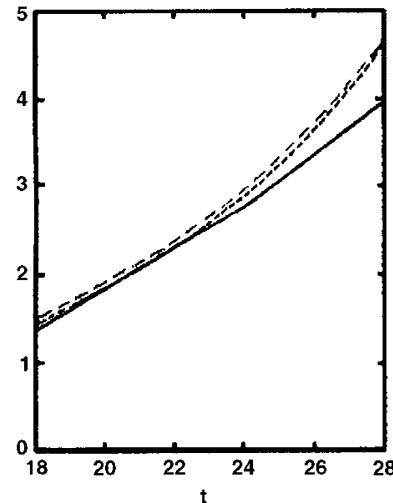

- experimental data

-- Cell Cycle rate distribution mode

Smith-Martin model

Figure 7 Dependences of (a) the mean clone size and (b) variance of the mean clone size in the model of continuous cell-cycle rate distribution (with the optimal parameters $\alpha=0.105$ and $\alpha_{\max }=0.109$ dash curve) and model of Smith-Martin (with the optimal parameters $T_{0}=6.8 \mathrm{~h}$ and $\gamma=0.331 / \mathrm{h}$ small dash curve). The experimental points are connected with straight lines. (a) Both approximations are equally good. (b) The dependence of variance of the mean clone size in the model of continuous cell-cycle rate distribution better fit the noisy experimental data than does the model of Smith-Martin. 
dependence $\exp (-\gamma T)$, where $1 / \gamma$ is a characteristic time of the stochastic transition, localized to G1, postulated in the model (Smith and Martin, 1973). Using the above equations for this case, we obtain the following equations for $N_{1}$ and $N_{2}$ for the model of Smith-Martin

$$
\begin{aligned}
& N_{1}(t)=\int_{0}^{\infty} \exp \left(\left(t-t_{0}\right) /\left(T+T_{0}\right)\right) \exp (-\gamma T) \mathrm{d} T \\
& N_{2}(t)=\int_{0}^{\infty}\left(N_{1}(t)-\exp \left(t-t_{0} /\left(T+T_{0}\right)\right)^{2} \exp (-\gamma T) \mathrm{d} T\right.
\end{aligned}
$$

where $t_{0}$ is the time point at which the mean clone size equals unity (see Table 1).

In this case, the minimal duration of cell cycle $T_{0}$ and the parameter $\gamma$, characterizing the rate of stochastic transition, are fitted parameters. These parameters are similar to the parameters $\alpha$ and $\alpha_{\max }$ in the model of a truncated normal distribution (in our model of a continuous cell-cycle rate distribution above). The integration in the formulas is performed over the parameter $T$ characterizing cells, proceeding to the postulated transition at a time $T$. Fitting of the mean clone size versus time and mean square clone size versus time curves, using both the model of a truncated normal distribution and that of Smith-Martin (with the optimal parameters $T_{0}=6.8 \mathrm{~h} \gamma=0.33 \mathrm{l} / \mathrm{h}$ ) to the experimental plots for the overall imaginal disc is shown in Figure $7 \mathrm{a}$ and $b$. The deterministic model with the distributed cellcycle rate has a better $\chi^{2}$ agreement with the experimental data than the Smith and Martin transition probability model.

\section{Heat shock}

The dependence of mitotic index of the wing imaginal disc cells on the time period between the heat shock and chemical fixation of the disc is shown in Figure 8. A drastic decrease in the mitotic index during $2-8 \mathrm{~h}$, its elevation during $12-16 \mathrm{~h}$, and stabilization during $16-28 \mathrm{~h}$ are evident. Thus, heat-shock results in a transient slowdown of the cell cycle, presumably while progressing from the stage G1 to $S$, although the data obtained fail to determine unambiguously the checkpoint underlying this retardation.

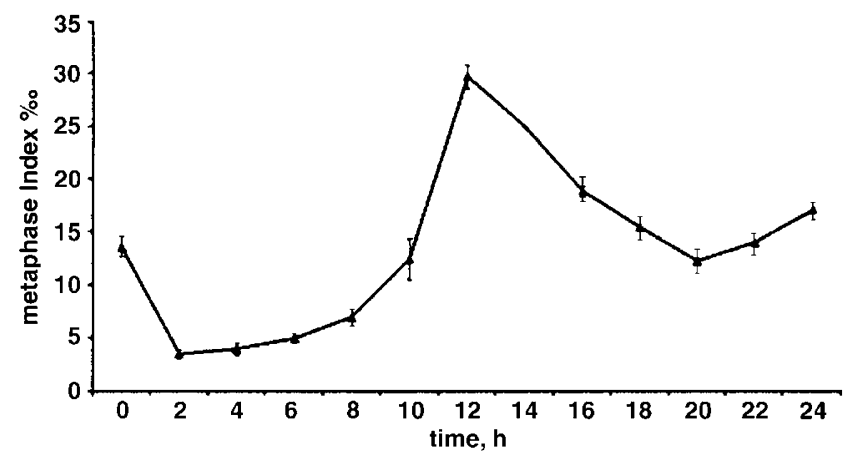

Figure 8 Relaxation of mitotic index in wing imaginal disc cells after heat shock: the ordinate, mean number of metaphases per 1000 interphase cells; the abscissa, time after heat shock. If the experimental error is below the triangle dimension, only the triangle is shown.

\section{Discussion}

Mosaic clones are traditionally used as a tool allowing cell proliferation to be studied. Recording of the shape, size, and position of mosaic clones on adult cuticle resulted in the discovery of the proliferation centers in wing imaginal disc and their topographical localization in intervein regions (Gonzalez-Gaitan et al, 1994). However, the time of clone induction in this approach is considerably earlier than the time when these clones are observed, preventing any detailed study of clone proliferation. In this work, we used the mosaic clones induced by excision of the FRT cassette, which, in turn, resulted in the marking of a particular cell and its progeny with $\beta$-galactosidase activity. This approach allows the moments of clone induction and of clone observation to be made considerably closer together in time.

The distribution of mosaic patches over the wing imaginal disc surface is uneven. We discovered nonrandom clusters of clones in the notum region. However, the anticipated (Gonzalez-Gaitan et al, 1994) intervein proliferation centers of the wing were not found. We believe this to be a consequence of the extremely small size of these intervein centers at the developmental stage under study.

The proliferation centers discovered are adjacent to regions of nonrandomly slow proliferation. On the map of wing imaginal disc presumptive rudiments, pairs consisting of a proliferation center next to a region of slow proliferation are localized to the regions displaying the most pronounced angular displacement in the course of development. Presumably, the function of the gradients of relative growth observed is in the creation of these displacements.

The analysis of the growth curves of average-sized clones over the entire disc allowed us to determine the average cell-cycle duration in this organ, which was $9.6 \mathrm{~h}$. A value of $8.5 \mathrm{~h}$ was found in earlier work analyzing the growth rate of mosaic clones recorded on the adult cuticle (Garcia-Bellido and Merriam, 1971). This discrepancy may be explained by the averaging of the cell-cycle duration over many developmental stages, used by Garcia-Bellido and Merriam, whereas, in our approach, the cell-cycle duration is averaged over the last $10 \mathrm{~h}$ of the third larval instar, which is close to the duration of one cycle. On the other hand, the duration of cell cycle assessed based on mosaic clone growth rates induced in the wing imaginal disc $48 \mathrm{~h}$ AED (after egg deposition) and recorded $120 \mathrm{~h}$ AED (Jonston et al, 1999; Datar et al, 2000) amounted to 12.9 and $12 \mathrm{~h}$, respectively. In these cases, the heat-shock duration used (1.5h) was three-fold longer than the heat shock we applied. Hence, the 12-h arrest of the cell cycle we noted (Table 1, Figure 8) would have taken place in these experiments. The correction of the data reported in Jonston et al (1999) by the inclusion of this period makes the cell-cycle duration estimated from their data equal to 9.7, which almost exactly coincides with our result.

In an earlier study, we found the cell-cycle duration in neural ganglia of third instar larvae to be $9 \mathrm{~h}$ using the technique of labeled mitoses (Trunova et al, 1998); this duration is close to the estimate obtained in this work. The duration of cell cycle in leg imaginal disc determined $96 \mathrm{~h}$ AED amounts to $7 \mathrm{~h}$ (Sustar and Schubiger, 2002) 
and, at later stages, $12 \mathrm{~h}$. As the developmental period studied (96h AED) corresponds to the end of the time period considered in this work, a cell-cycle duration of 9.6 is not inconsistent with these data.

According to the data listed in Table 1, the mean cellcycle duration, amounting to $9-10 \mathrm{~h}$, varies in a narrow range. However, the proliferation centers PR2 and PR3 display shorter cell cycles: 8.1 and $8.8 \mathrm{~h}$, respectively. The observed clustering of quickly dividing clones in the proliferation center PR2 agrees well with the cell cycle actually being shortened in this region. On the other hand, the center PR1 was found as the region displaying a clustering of dividing cells; however, no cell cycle shortening or clustering of quickly dividing cells was observed. These features of PR1 may indicate that not only a shortening of the cell cycle but possibly also the recruitment of previously nondividing cells into the cell cycle are involved in the regulation of the cell proliferation rate in proliferation centers.

Smith and Martin proposed a transition probability model through analyzing the data from time-lapse cinematography of normal and transformed cells in cell culture (Smith and Martin, 1973). This model implies the presence of additional stochastic phase in G1 along with the deterministic phase corresponding to $S, G 2, M$, and the remaining part of G1. The deterministic phase corresponds to a constant rate of progression of the cells through the cell cycle, whereas the stochastic phase is described as a monomolecular reaction in chemical kinetics, that is, the probability of a cell not having left the stochastic phase decreases exponentially with time. Smith and Martin demonstrated that it was the stochastic phase that determined the dispersion of cell-cycle duration, whereas the variation in the rate of deterministic phase progress amounts to only $10 \%$ of its duration (Smith and Martin, 1973).

Smith and Martin model considered here has sound experimental confirmation (Robinson et al, 1976; Shields, 1978; Shields et al, 1978; Ronning and Seglen, 1982; Rabinovich, 1983) and served as a basis for theoretical models describing the curves of labeled mitoses (Cain and Chau, 1997). We have demonstrated that the model of Smith and Martin is less accurate in describing our experimental data than is our model ascribing the major role in the heterogeneity of proliferation rates to the dispersion of the rates of cell-cycle progression in a deterministic phase. This means that either the phenomenon of stochastic phase is limited to cell culture or this phase is described by a set of constants rather than by one constant.

\section{Acknowledgements}

The work was supported by Russian Foundation for Basic Research (Grant No. 02-04-49323).

\section{References}

Bryant PJ (1975). Pattern formation in the imaginal wing disc of Drosophila melanogaster: fate map, regeneration and duplication. J Exp Zool 193: 49-77.

Cain SJ, Chau PC (1997). Transition probability cell cycle model. Part I. Balanced growth. J Theor Biol 185: 55-67.
Datar SA, Cobs HWJ, Crus AFA, Lehner CF, Edgar BA (2000). The Drosophila cyclin D-Cdk4 complex promotes cellular growth. EMBO J 19: 4543-4554.

Garcia-Bellido A, Marriam JR (1969). Cell lineage of the imaginal discs in Drosophila gynandromorphs. J Exp Zool 170: $61-75$.

Garcia-Bellido A, Merriam JR (1971). Parameters of the wing imaginal disc development of Drosophila melanogaster. Dev Biol 24: 61-87.

Garcia-Bellido A, Ripoll P, Morata G (1973). Developmental compartmentalization of the wing disc of Drosophila. Nat $N$ Biol 245: 251-253.

Gell JD, Pardue ML (1971). Nucleic acid hybridization in cytological preparations. Methods Enzymol 21: 470-480.

Gonzalez-Gaitan M, Capdevila M, Garcia-Bellido A (1994). Cell proliferation in the wing imaginal disc of Drosophila. Mech Dev 40: 183-200.

Johnston LA, Edgar B. (1998). Wingless and notch regulate cellcycle arrest in the developing Drosophila wing. Nature 394: 82-84.

Jonston LA, Prober DA, Edgar BA, Eisenman RN, Gallant P (1999). Drosophila myc regulates cellular growth during development. Cell 98: 779-790.

Milan M, Campuzano S, Garcia-Bellido A (1996a). Cell cycling and patterned cell proliferation in the wing primordium of Drosophila. Proc Natl Acad Sci USA 93: 640-645.

Milan M, Campuzan S, Garcia-Bellido A (1996b). Cell cycling and patterned cell proliferation in the wing during metamorphosis. Proc Natl Acad Sci USA 93: 11687-11692.

Modollel J, Campuzano S (1998). The achaete-scute complex as an integrating device. Int I Dev Biol 42: 275-282.

O'Brochta DA, Bryant P (1985). A zone of non-proliferating cells at a lineage restriction boundary in Drosophila. Nature $\mathbf{3 1 3}$ 138-141.

Omelyanchuk LV, Volkova EI, Fedorova SA (1997). Characterization of insertion mutations leading to mitotic abnormalities in Drosophila melanogaster by means of the reporter genecontaining transposon. Genetika 33: 1494-1501.

Rabinovich PS (1983). Regulation of human fibroblast growth rate by both noncycling cell fraction transition probability is shown by growth in 5-bromodeoxyuridine followed by Hoechst 33258 flow cytometry. Proc Natl Acad Sci USA 80: 2951-2955.

Robinson JH, Smith JA, Totty NF, Riddle PN (1976). Transition probability and the hormonal and density-dependent regulation of cell proliferation. Nature 262: 298-300.

Ronning OW, Seglen PO (1982). The relation between protein accumulation and cell cycle traverse of human NHIK 3025 cells in unbalanced growth. J Cell Physiol 112: 19-26.

Shields R (1978). Further evidence for random transition in the cell cycle. Nature 273: 755-758.

Shields R, Brooks RF, Riddle PN, Capellaro DF, Delia D (1978). Cell size, cell cycle and transition probability in mouse fibroblasts. Cell 15: 469-474.

Smith JA, Martin L (1973). Do cells cycle? Proc Natl Acad Sci USA 70: 1263-1267.

Struhl G, Basler K (1993). Organizing activity of wingless protein in Drosophila. Cell 72: 527-540.

Sustar A, Schubiger G (2002). Does regenerative growth increase cell potency in imaginal disc? A Dros Res Conf 43: 178A.

Trunova SA, Dubatolova TD, Omelyanchuk LV (1998). Determination of the expression of $\mathrm{chb}^{\mathrm{v} 40}$ gene in the cell cycle. Genetika 29: 342-346.

Tsuji H, Tobari I (1980). Effects of 5-bromodeoxyuridine on metamorphosis and cell cycle kinetics of ganglion cells in Drosophila melanogaster. Exp Cell Res 125: 79-85. 